\title{
High Performance Infrared Gas Measurement System Based on a Semiconductor Gas Sensor IR Source with Tunable Fabry-Pérot Filter
}

\author{
Martin Leidinger ${ }^{1}$, Eliseo Pignanelli, ${ }^{1,2}$, Karsten Kühn ${ }^{1}$, Tilman Sauerwald ${ }^{1}$, Andreas Schütze ${ }^{1}$ \\ ${ }^{1}$ Laboratory for Measurement Technology, Saarland University, Saarbrücken, Germany, \\ s9mnleid@stud.uni-saarland.de \\ ${ }^{2}$ ZeMA - Centre for Mechatronics and Automation GmbH, Saarbrücken, Germany
}

\begin{abstract}
In this work, a miniature infrared (IR) spectrometer for gas analysis was realized by using a microstructured Fabry-Pérot interferometer as a tunable filter. Two of these filters were applied for two different wavelength ranges ( 3.9 to $5.0 \mu \mathrm{m}$ and 8 to $10.5 \mu \mathrm{m}$, respectively). Thus, a wide range of gases can be detected and quantified. A semiconductor gas sensor was used as IR radiation source. With filter modulation frequencies of several $\mathrm{Hz}$, optical spectra can be recorded quickly and several gases can be monitored simultaneously. Furthermore, a computer model of the system was created which describes all the steps of the measurement chain and calculates the influences on the measurement at each step. To generate the models of the Fabry-Pérot filters (FPF), for example, the devices were characterized using Fourier Transform Infrared (FTIR) spectroscopy. Gas measurements were performed in the two wavelength ranges to demonstrate the functionality of the system's operating mode as a low cost spectrometer.
\end{abstract}

Key words: Infrared transmission spectroscopy, gas analysis, Fabry-Pérot filter (FPF), metal oxide (MOX) semiconductor gas sensor, miniature infrared (IR) spectrometer.

\section{Introduction}

A widely used method for gas measurements is infrared radiation absorption analysis. This optical method is based on the absorption of infrared light at gas specific wavelengths [1]. Selectivity in an IR measurement system can be achieved by using a broad band IR radiation source and a narrow band wavelength filter tailored to the target gas [2]. However, if such a system is to be used to detect more than one gas, several different filters or detectors are necessary, which increases the number of components and thus the complexity and the price of the system. More versatile optical gas analysis systems can monitor a wavelength range by varying either the emitted radiation or the detected radiation, e.g. with an interferometer [3]. Such a spectrometer can be created by using a tunable Fabry-Pérot wavelength filter [4], [5]. Micromachined Fabry-Pérot interferometers are available for low power and low cost integrated solutions [6].

IR spectroscopy is capable of detecting a wide range of gases, but some gases are not IR active $\left(\right.$ e.g. $\left.\mathrm{H}_{2}\right)$ and the detection limit is quite high (depending on optical path length).
Another method to detect and measure gases is the use of semiconductor (sc) gas sensors, which change their electric conductivity in the presence of reducing and oxidizing gases. Semiconductor gas sensors are highly sensitive to a great variety of gases and, using multisignal methods like temperature cycling, they can provide a selective signal [7], [8]. However, they cannot detect inert gases (like $\mathrm{CO}_{2}$ ) and they also show a limited accuracy for quantification. For some applications, a combined system is useful. For example, monitoring indoor air quality needs the assessment of some hundred ppm of $\mathrm{CO}_{2}$ and of ppb concentrations of toxic volatile organic compounds [9]. Since sc gas sensors usually are heated with an internal heater structure to several hundred degrees Celsius, they can be used as IR radiation sources. Two types of semiconductor (metal oxide, MOX) gas sensors are available: silicon based microstructured sensors and ceramic based sensors. The microstructured sensors are low power devices, they have small heated areas and masses and are heated to approx. $400^{\circ} \mathrm{C}$. The suitability of these sensors as IR radiation sources for spectroscopy has been analyzed in a previous work [10]. Their small thermal masses allow for 
a modulation frequency of up to $100 \mathrm{~Hz}$ in order to generate periodic measurement signals. The ceramic based sensors operate at much higher heater power (> 1W) and higher temperatures of up to $800^{\circ} \mathrm{C}$. This means they emit IR radiation at considerably higher intensity than the microstructured sensors and can be used as more powerful IR radiation sources for spectrometry. In this paper, we focus on IR gas analysis without discussing MOX sensor signals.

\section{Experimental}

The measurement system used in this work (Fig. 1) combines the features of the systems used in [5] (tunable filter) and [10] (MOX gas sensor as IR source). It contains a ceramic substrate MOX gas sensor (GGS1330, Umweltsensortechnik, Geschwenda, Germany) as IR radiation source as well as a tunable FabryPérot filter (TFP3950 for 3.9 to $5.0 \mu \mathrm{m}$ or TFP80105 for 8 to $10.5 \mu \mathrm{m}$, InfraTec, Dresden, Germany). A thermopile sensor (HIS A21 Fx G5600, Heimann Sensor, Dresden, Germany), with broadband filter suitable for the observed wavelength range detects the transmitted radiation.

Additional components of the system are sensors to monitor the ambient conditions

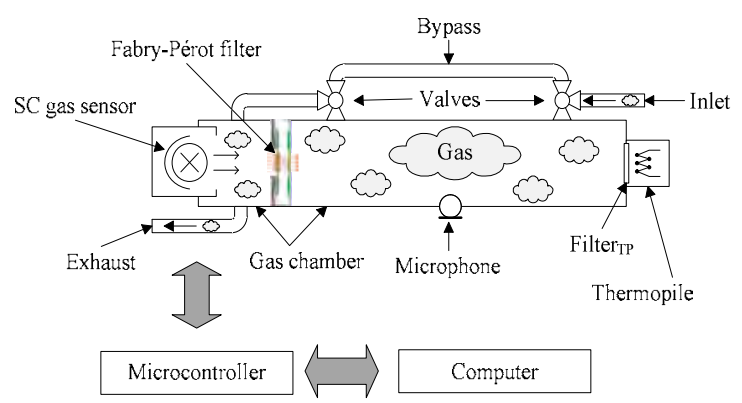

Fig. 1: Overview of the combined semiconductor/IR gas measurement system and its components

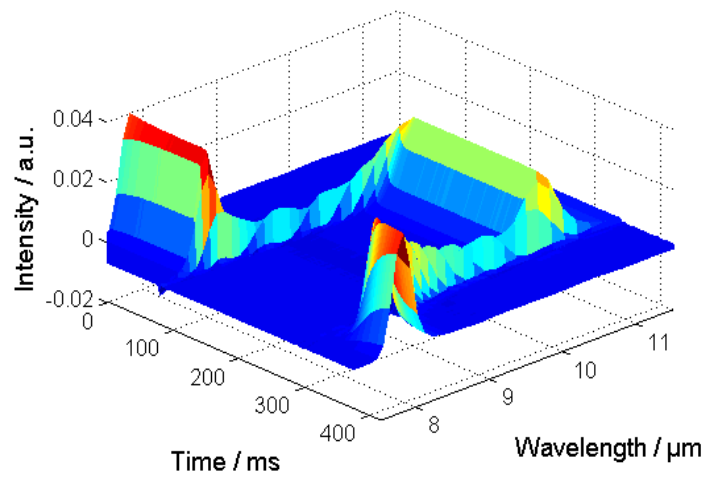

Fig. 2: Time response of Fabry-Pérot filter TFP80105 at switch from maximum to minimum wavelength, $3 D$ plot (pressure, temperature, relative humidity), a microphone for photoacoustic spectrometry measurements (PAS, not used in this work) and valves to open/close the measurement chamber. All these components are attached to the IR chamber, which has an optical path length of $9 \mathrm{~cm}$. The sensors and actuators are connected to a microcontroller board which controls the operation of the system. The analog sensor signals are recorded by the microcontroller's internal analog to digital converter (ADC, 10bit resolution) at a sampling rate of $333 \mathrm{~Hz}$. The controller communicates to a host PC via USB. Configuration of the system, data acquisition and online signal processing on the $\mathrm{PC}$ are handled in a LabVIEW graphical user interface.

With the 'stepscan' mode of an FTIR spectrometer (Vertex 80v, Bruker) the dynamic behavior of the tunable filters was recorded. Fig. 2 shows the 3D data of the measurement of the TFP80105 filter. The filter was switched between its maximum and minimum wavelengths (10.45 to $7.8 \mu \mathrm{m}, 0$ to $50 \mathrm{~V}$ ) at a frequency of $2.5 \mathrm{~Hz}$. Fig. 3 shows the projected view of the measurement on the time/wavelength plane, the signal intensity is indicated by the color. From this data, time constants of the switching process were extracted and the maximum modulation frequencies for the filters were obtained. They differ significantly for the two filters, at up to $8 \mathrm{~Hz}$ for the TFP80105 and $4 \mathrm{~Hz}$ for the TFP3950.

The adjustable parameters for the FPF modulation operating mode are the temperature of the gas sensor heater, the FPF modulation frequency, the covered wavelength range, the number of filter steps within a cycle and the shape of the filter modulation. The nominal center wavelength (CWL) of the FPF can be tuned either linearly or sinus-shaped. Other modulation shapes can also be programmed.

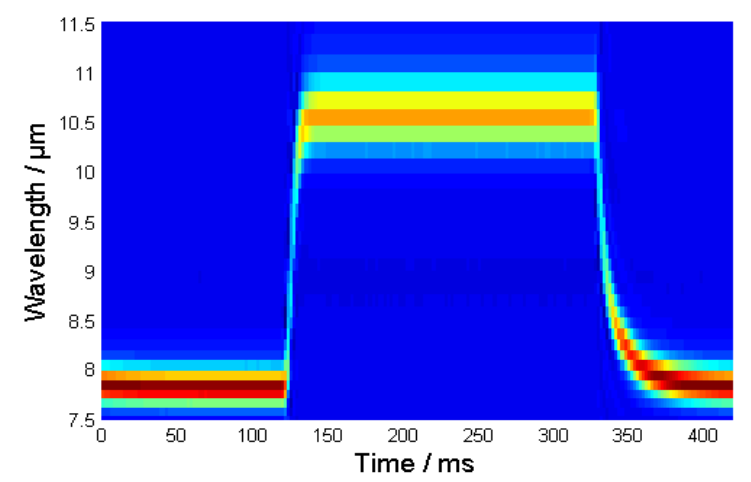

Fig. 3: Time response of Fabry-Pérot filter TFP80105 at switch from maximum to minimum wavelength, projected $2 D$ plot 


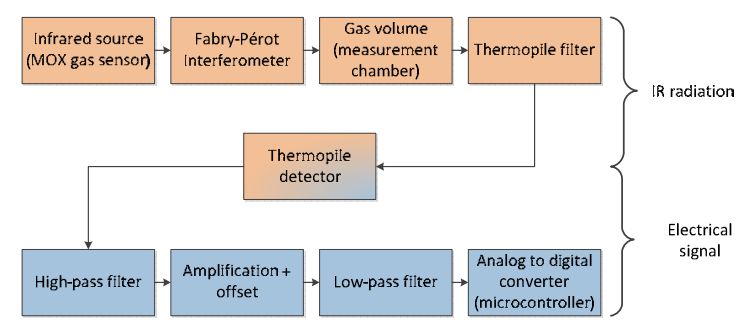

Fig. 4: Step-by-step flow-chart of the different independent influences on the IR radiation and the electrical signal in the gas measurement system.

\section{Modeling of the system}

A computer model for the measurement was created in Matlab. It contains a model for each step of the measurement and calculates the influence on either the infrared radiation or the electrical signal. The model was created in order to estimate measurement results and optimize the system's settings concerning the filter modulation. Therefore, essentially the different independent influences on the IR radiation and the electrical thermopile sensor signal, as shown in Fig. 4, were modeled to obtain a qualitative prediction of the measured signal. The approach will be presented here for the UST GGS1330 gas sensor, the TFP3950 filter and $\mathrm{CO}_{2}$ as the absorbing gas. The first step is the emission of infrared radiation of the MOX gas sensor. The spectral emission of the UST GGS1330 was measured for several temperatures with the FTIR spectrometer, the result is shown in Fig. 5. The small peaks at $4.2-4.3 \mu \mathrm{m}$ result from absorption of the IR radiation due to $\mathrm{CO}_{2}$ from the ambient air inside the spectrometer.

Next, the influence of the Fabry-Pérot filter must be analyzed. The filter was characterized with FTIR measurements as well, see Fig. 6. From this data, features were extracted which allow a simple modeling of the transmission curve for

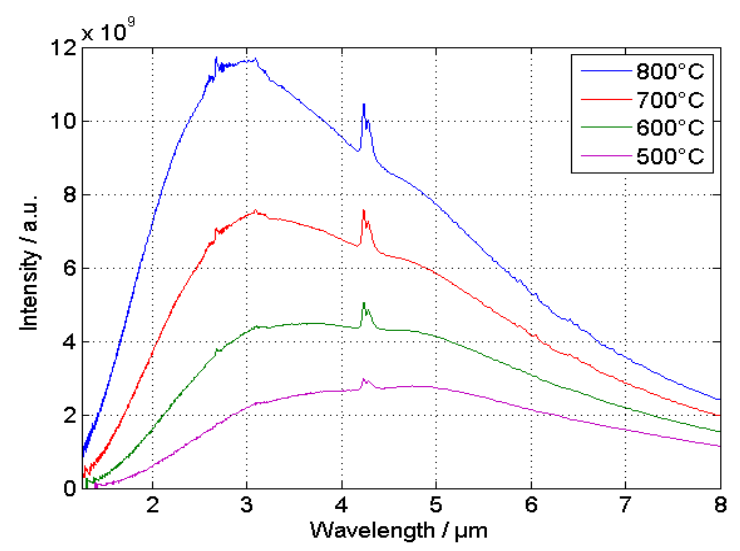

Fig. 5: FTIR measurements of the emitted infrared spectrum of UST GGS1330 at different heater temperatures

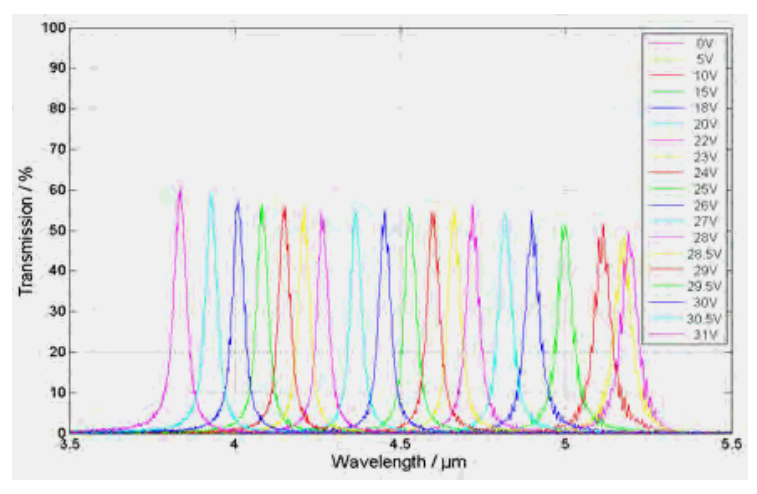

Fig. 6: Measured FTIR transmission spectra of the TFP3950 filter at different control voltages

any selected center wavelength. Fit functions for the CWL (signal maxima) and the curves' full width half maximum (FWHM) values were determined and can be used to compute a Gaussian function transmission peak at any FPF center wavelength. In the next step these spectra are multiplied by the emission spectrum of the heater to take into account the uneven IR intensity of the source over the wavelength range from 3.5 to $5.5 \mu \mathrm{m}$, see Fig. 5. The following influence is the absorption of radiation by the gas. The gas transmission spectrum is calculated from the HITRAN (High Resolution Transmission) database data according to [11]. Fig. 7 shows the transmission spectrum of $\mathrm{CO}_{2}$ at a concentration of $400 \mathrm{ppm}$ in the selected wavelength range. Each of the modeled FPF transmission curves is multiplied by the gas transmission spectrum to determine the IR intensity for each FPF center wavelength after the radiation has passed through the gas in the measurement chamber. Modeling of the thermopile detector is simply an integration of the radiation spectra for each examined CWL obtained from the last step. Fig. 8 shows the calculated thermopile signal for each wavelength of the selected FPF range for pure air (red) and for a $\mathrm{CO}_{2}$ concentration of $400 \mathrm{ppm}$ in

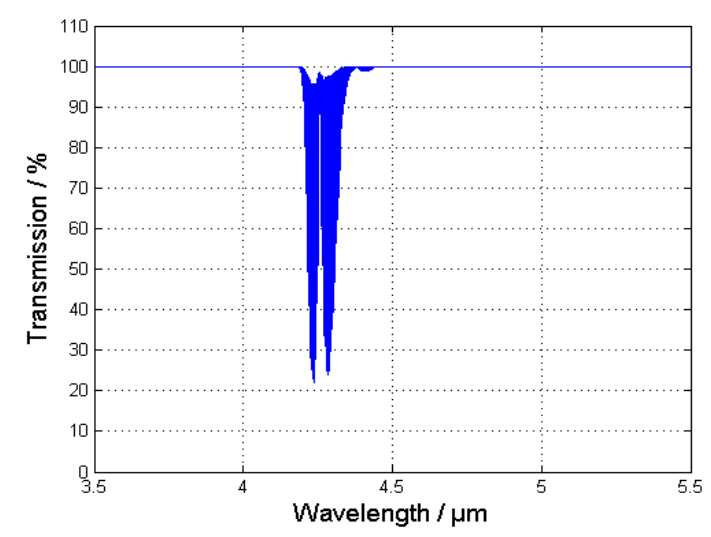

Fig. 7: Calculated transmission spectrum of $\mathrm{CO} 2$ over the wavelength range of the TFP3950 filter, concentration $400 \mathrm{ppm}$ 


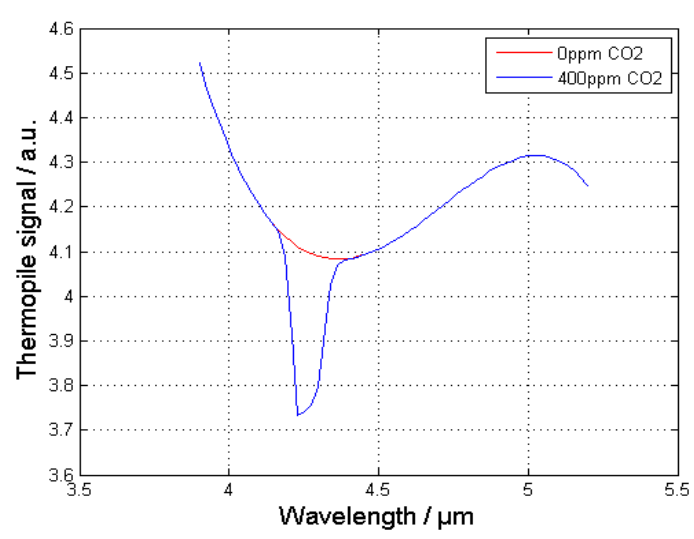

Fig. 8: Calculated thermopile sensor signal for every adjustable center wavelength of the TFP3950 filter at 0 ppm CO2 and 400 ppm CO2

air (blue).

With this relation of signal and wavelength, the estimated thermopile signal vs. time can be calculated for a full cycle of any selected wavelength modulation of the filter. Several cycles are strung together to generate the complete thermopile signal for FPF modulation. This calculated sensor signal is then fed into the models of the electrical signal conditioning (cf. Fig. 4): First it passes a first order high pass filter to filter out any DC components. Then it is amplified to generate a larger signal. An offset of $+2.5 \mathrm{~V}$ is added in order to shift the signal, which oscillates around $0 \mathrm{~V}$ behind the high pass filter, into the center of the $5 \mathrm{~V}$ voltage range of the microcontroller's ADC. The last influence on the signal is a first order low-pass filter which filters any high frequency noise. The final amplified and filtered calculated thermopile signal is shown in Fig. 9.

A real measurement with the same system parameters was performed and compared to the modeled signal. One full cycle of this measurement is shown in Fig. 10. The basic shapes of the modeled and the real curves are very similar, the gas absorption, however, is not

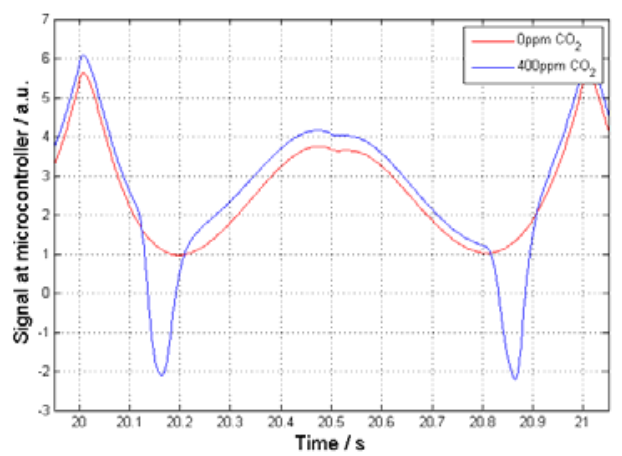

Fig. 9: One modeled cycle of the IR detector signal after electrical conditioning (high-pass filter, amplification, low-pass filter) as prominent in the measurement as in the calculated signal. Still, it shows that the Matlab model constitutes a useful tool to describe the system's behavior. In practical applications, signals would be acquired over several periods and evaluated with suitable methods, e.g. lockin-method or FFT. A trade-off between acquisition time, i.e. number of cycles, and resolution, i.e. noise level, could be selected depending on application requirements.

\section{Demonstration spectroscopy measurement}

To demonstrate the FPF modulation mode in the higher wavelength range of the TFP80105 filter, saturated vapors of acetone and methanol were used as test gases. The vapor pressure and corresponding concentrations at a temperature of $22{ }^{\circ} \mathrm{C}$ are $267.5 \mathrm{mbar} / 26.75 \%$ for acetone and $146.6 \mathrm{mbar} / 14.66 \%$ for methanol [12]. The measurements were performed with the GGS1330 sensor at $700^{\circ} \mathrm{C}$ as IR radiation source, the FPF80105 was used at a modulation frequency of $1 \mathrm{~Hz}$ in a wavelength range from 8.2 to $10.4 \mu \mathrm{m}$. Sets of 20 consecutive FPF cycles were acquired and processed. Fig. 12 shows a section of four cycles of the acquired IR signal. The first two cycles show the signal without any absorbing gas present. The signal form is determined by the change of transmission characteristics over the wavelength range of the Fabry-Pérot filter. There is a conspicuous shift visible between the nominal wavelength and the infrared signal. This is mostly because the FPF lags behind the control voltage by a few milliseconds. The thermopile also has a part in this effect because it has a time constant of approx. $8 \mathrm{~ms}$ [13]. This shift must be taken into account in the analysis of the data.

In the third cycle, the absorbing gas, acetone, has flooded the measurement chamber, the drop in the IR signal is clearly visible at the lowest FPF wavelength at second 40.

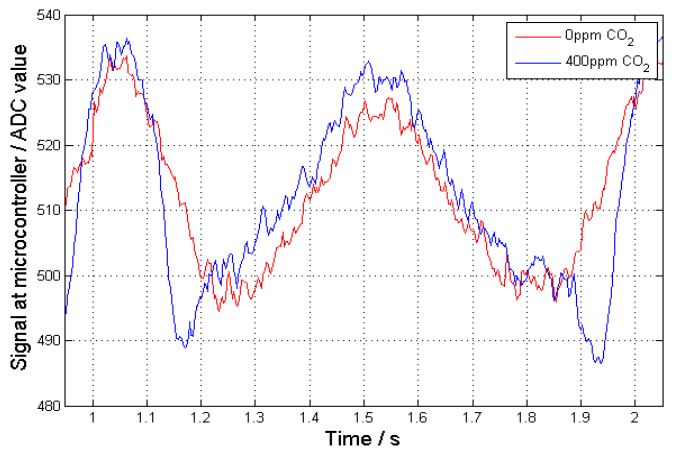

Fig. 10: Measured signal of the previously modeled measurement of 0 ppm CO2 and 400 ppm CO2, see Fig. 9 


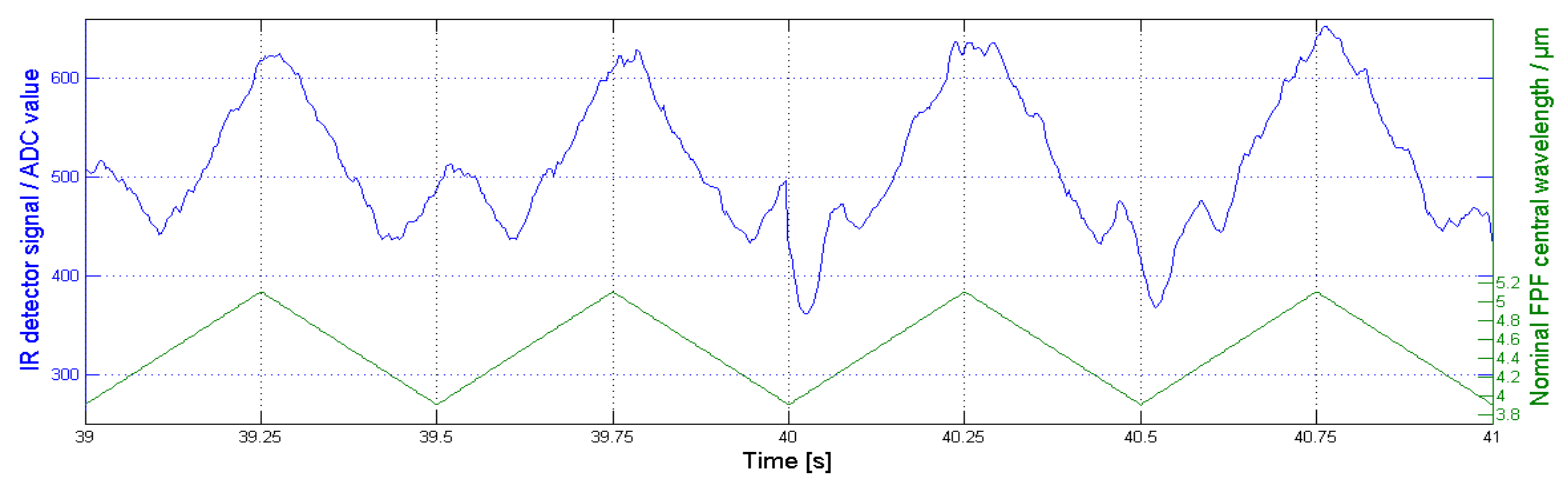

Fig. 11: Gas response of normalized IR detector signal. IR signals are divided by reference signal and then averaged over 20 cycles (blue); nominal center wavelength of the Fabry-Pérot filter (green)

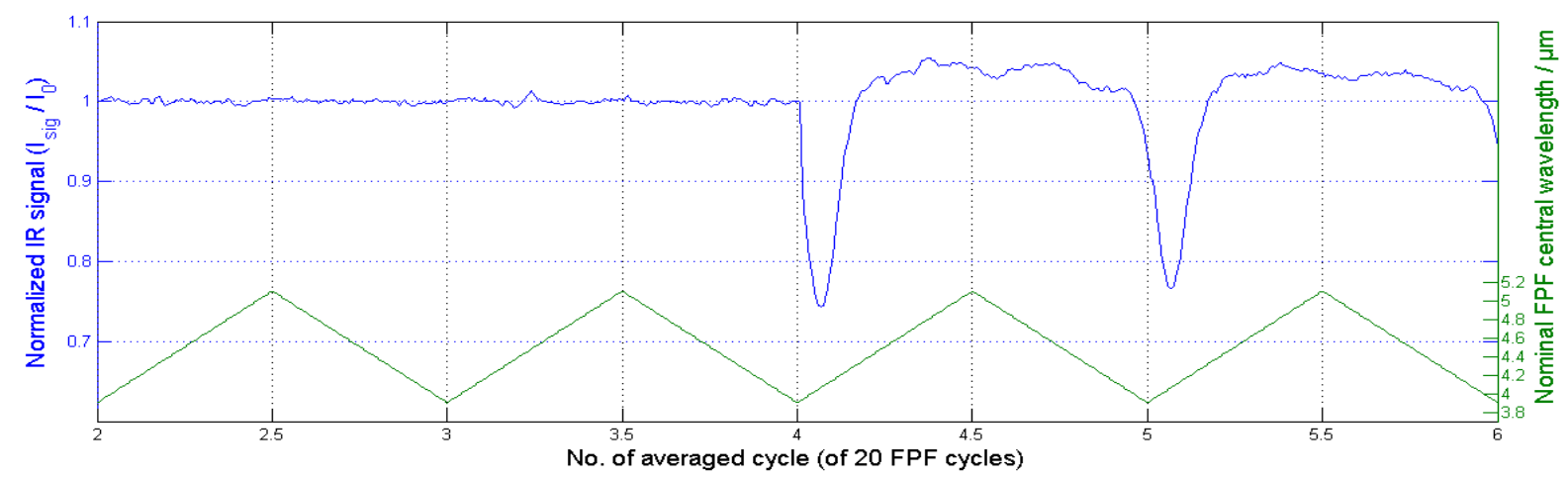

Fig. 12: Infrared detector signals while flooding the measurement chamber with the acetone gas (blue), nominal center wavelength of the Fabry-Pérot filter (green)

At the beginning of a measurement, before the absorbing gas is introduced into the measurement chamber, the signal over one IR cycle is acquired and saved as reference. The subsequently acquired signal sets are then divided by this reference in order to suppress the zero signal waveform without absorption. Several normalized cycles are averaged in order to get one averaged cycle from each IR signal set. This data is shown in Fig. 11. It is clearly visible that the influence of the gas began in the fourth set of cycles. The signal values can go above 1 here because of the electrical high-pass filtering of the thermopile signal, which shifts the high parts of the signal up a bit, see also Fig. 12.

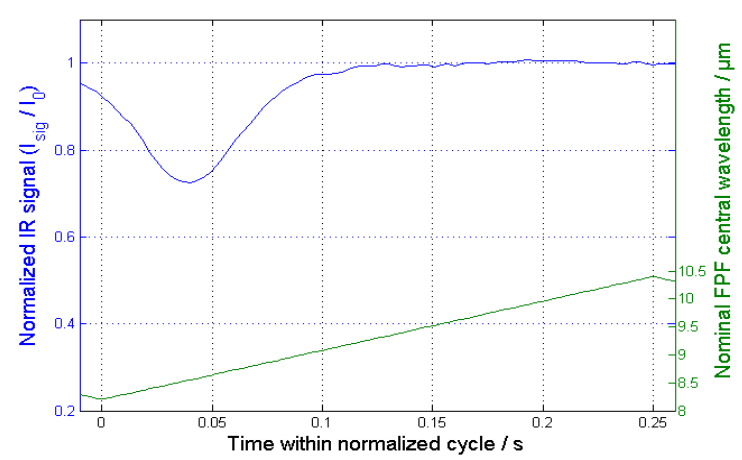

Fig. 13: Normalized and averaged IR signal of one sweep of the wavelength range for acetone
Fig. 13 - Fig. 15 show normalized and averaged signals over half a cycle, i.e. one sweep of the covered spectrum of the Fabry-Pérot filter, with different test gases inside the measurement chamber. Fig. 13 shows the spectrum of the acetone measurement. Taking into account the signal shift explained earlier (about $30 \mathrm{~ms}$ ), the absorption is visible at the lower limit of the wavelength range at $8.2 \mu \mathrm{m}$, as expected [12]. The signal peak is quite narrow because not the whole absorption peak of the gas is covered by the filter. The result of the measurement with methanol vapor is shown in Fig. 14. Adjusted by the signal shift, the center of the absorption peak is located at approx. $9.8 \mu \mathrm{m}$, corres-

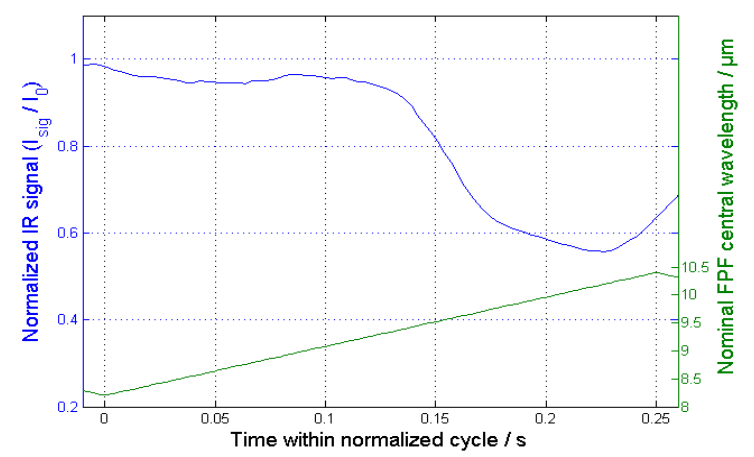

Fig. 14: Normalized and averaged IR signal of one sweep of the wavelength range for methanol 


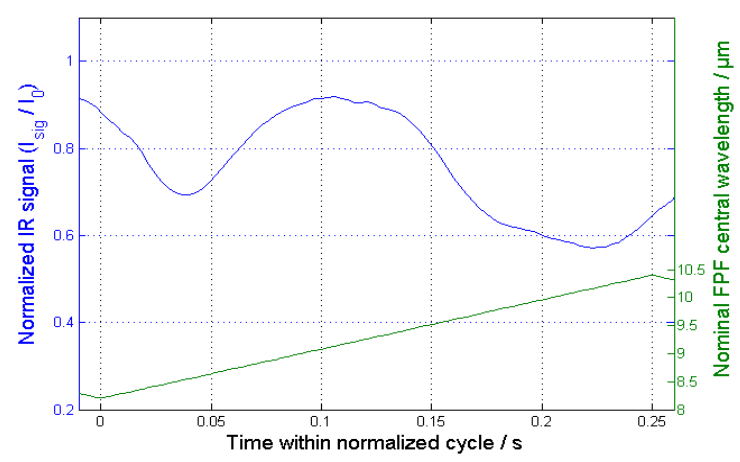

Fig. 15: Normalized and averaged IR signal of one sweep of the wavelength range for a mixture of acetone and methanol

ponding to the two absorption peaks of methanol at 9.5 and $10 \mu \mathrm{m}$, respectively [12]. The width of the filter transmission is too high to resolve the peaks individually, although an indication of two peaks can be seen. In a final measurement, both gases were introduced into the measurement chamber together. Fig. 15 shows the infrared spectrum with both gases. The two absorption peaks are clearly distinguished allowing simultaneous identification and quantification of both gases.

\section{Conclusion and outlook}

In this work, a new operating mode for a gas measurement system was implemented and tested. It comprises a periodic modulation of a micromachined Fabry-Pérot interferometer in order to create a miniature IR spectrometer. A constantly heated semiconductor gas sensor was used as the IR radiation source.

The system and the different influences on the gas measurement were modeled in Matlab in order to generate a quick and easy way to determine suitable system parameters for gas measurements. The general validity of the model was checked through a test measurement.

Infrared radiation transmission measurements were performed in the wavelength range of 8.2 to $10.4 \mu \mathrm{m}$ to show the capability of the system to simultaneously detect, identify and quantify gases absorbing IR radiation in this range.

In the future, the new operating mode will be used in combination with temperature cycled operation of the semiconductor gas sensor. With this combination, the number of detectable gases can be increased considerably since different gases and gas concentrations can be measured using IR spectroscopy and MOX sensors, respectively. For gases that react to both principles, the MOX gas sensor could be used to detect small concentrations while the IR measurement quantifies high concentrations. In concentration ranges that both methods can resolve, a redundancy in the measurements would be available, allowing an inherent selfmonitoring of the system.

\section{References}

[1] H.W. Siesler, Y. Ozaki, S. Kawata, H.M. Heise, "Near-Infrared Spectroscopy", Wiley-VCH, 2002, pp. 22-41

[2] P.T. Moseley, J.O.W. Norris, D.E. Williams, "Techniques and Mechanisms in Gas Sensing", IOP Publishing, 1991, p. 251

[3] H. Günzler, H. Gremlich,"IR Spektroskopie“, Issue 4, Wiley VCH, 2003, pp. 39-42

[4] M. Ebermann, N. Neumann, K. Hiller, M. Meinig, "Spectrally Tunable IR-Sensors for Industrial Production Metrology and Medical Gas Analysis," tm - Technisches Messen 10/2012 (in German), pp. 440 - 450.

[5] K. Kühn, M. Siegwart, E. Pignanelli, T. Sauerwald, A. Schütze, "Versatile Infrared Gas Measurement System with tunable Microstructured Fabry-Pérot Filter," Proc. IEEE IMTC, pp. 1938 - 1943 (2012)

[6] N. Neumann, M. Ebermann, S. Kurth, K. Hiller, "Tunable infrared detector with integrated micromachined Fabry-Perot filter", J. Micro/Nanolith. MEMS MOEMS. 7(2), 021004 (April 30, 2008)

[7] A.P. Lee, B.J. Reedy, "Temperature modulation in semiconductor gas sensing," Sensors and Actuators B 60 (1999), pp. 35-42

[8] A. Gramm, A. Schütze, "High performance solvent vapor identification with a two sensor array using temperature cycling and pattern classification," Sensors \& Actuators B 95 (2003) pp. $58-65$

[9] O. Kiesewetter, "Energieeinsparung durch bedarfsgerechtes Lüften”, ELMUG Technologiekonferenz elmug4future, Suhl, July 05-06, 2011

[10] K. Kühn, M. Leidinger, E. Pignanelli, A. Schütze, "Investigations on a MOX Gas Sensor as an Infrared Source for an IR-based Gas Sensing System," Proc. IMCS 2012, pp. 233 236 (2012)

[11] L.S. Rothman et al., "The HITRAN Molecular Spectroscopic Database and HAWKS (HITRAN Atmospheric Workstation): 1996 edition", Journal of Quantitative Spectroscopy and Radiative Transfer, Volume 60, Number 5, 1998

[12] NIST Chemistry Webbook database, http://webbook.nist.gov/chemistry/, accessed 22. January 2013

[13] Heimann Sensor, Datasheet Integrated Sensor Type HIS Ax1 Fx G5600, 2011 